Revue d'histoire de l'Amérique française

REVUE D.HISTOIRE DE L'AMÉRIQUE FRANÇAISE

\title{
La construction du fort Saint-Jean, 1748-1749
}

\section{Roland Lamontagne}

Volume 15, numéro 1, juin 1961

URI : https://id.erudit.org/iderudit/302093ar

DOI : https://doi.org/10.7202/302093ar

Aller au sommaire du numéro

Éditeur(s)

Institut d'histoire de l'Amérique française

ISSN

0035-2357 (imprimé)

1492-1383 (numérique)

Découvrir la revue

Citer cet article

Lamontagne, R. (1961). La construction du fort Saint-Jean, 1748-1749. Revue

d'histoire de l'Amérique française, 15(1), 35-40. https://doi.org/10.7202/302093ar d'utilisation que vous pouvez consulter en ligne.

https://apropos.erudit.org/fr/usagers/politique-dutilisation/ 


\title{
LA CONSTRUCTION DU FORT SAINT-JEAN
}

\author{
$1748-1749 *$
}

Vers la fin de la guerre de Succession d'Autriche, RolandMichel Barrin de La Galissonière décidait de construire le poste fortifié de Saint-Jean dans le double but de secourir le fort SaintFrédéric et de faciliter la colonisation agricole de la vallée du lac Champlain. L'aménagement d'une route reliant le fort SaintJean et Laprairie avait pour objet d'améliorer la communication entre le fort Saint-Frédéric et Montréal et d'économiser au chapitre des transports. ${ }^{1}$

Dans un mémoire «porté au Roy le 28 janvier 1748 », La Galissonière rendait compte des différents partis de guerre composés de Canadiens et de Sauvages qui, au cours de 1747, avaient effectué des opérations militaires dans les colonies britanniques. Il rappelait l'échec du chevalier de Niverville, «Enseigne en second d'une compagnie des troupes de la Colonie », qui en route vers Boston fut surpris par un détachement bien supérieur au

* L'auteur tient à exprimer sa gratitude à M. Pierre Brunet, adjoint de l'archiviste du Dominion, à M. J.-Antoine Pelletier, bibliothécaire adjoint, archives de la province de Québec, à M. J.-N. Rouleau, directeur du Département d'Histoire, Collège Militaire Royal de Saint-Jean, à M. Yves Laissus, archiviste-paléographe et bibliothécaire au Muséum national d'histoire naturelle, qui lui ont fait parvenir des renseignements et des photocopies de sources manuscrites émanant de Roland-Michel Barrin de La Galissonière.

$1 \ll$ Le fort du rapide $S^{\text {te }}$ Therese a été entierement abandonné: une partie des matériaux a servi a en construire un autre a $\mathrm{s}^{t}$ Jean au dessus de tous les rapides. Au moyen de ce fort et du chemin qui prend depuis ce fort jusqu'a la prairie de Lamagdeleine, celui de $S^{t}$ Frederic se trouve a portée d'etre secouru en 2 fois 24 heures, au lieu qu'auparavant on étoit obligé de faire passer les secours par Chambli, d'ou il falloit monter trois rapides, Chambli, $\mathbf{S}^{\text {te }}$ Therese, et $\mathbf{S}^{t} \mathrm{Jean}$; et par le calcul qu'ils ont fait il y aura $6000 £$ d'epargne tous les ans sur les transports. Ce nouveau fort facilitera les etablissemens des terres du Lac Champlain et les exploitations des bois de construction. 》 Canada, $\mathrm{M}^{\mathrm{rs}}$ de la Galissonière et Bigot, 26 septembre 1748. Envoyent un journal de ce qui s'est passé en Canada a l'occasion des mouvemens de guerre et des differens avis qu'on a reçu a Quebec depuis le mois de novembre 1747, AC, C11A, 91: 20-21; Sylvain Girerd, L'Oeuvre militaire de La Galissonière au Canada (Saint-Etienne, 1902), 38. 
sien. On avait appris la nouvelle des préparatifs en vue d'une attaque de frontière. François Pierre de Rigaud de Vaudreuil, major des Trois-Rivières, avait reçu la mission de se porter au secours du fort Saint-Frédéric à la tête d'environ 1,200 hommes. ${ }^{2}$

Le 28 mars 1748, Roland-Michel Barrin de La Galissonière avait formulé une ordonnance à l'adresse du Sieur de Léry qui devait se rendre à Saint-Jean en vue de diriger les travaux de construction. La phase préliminaire de cette mission comportait une visite au chantier de Corbin à la rivière Chazy « pour y prendre les eclaircissemens, secours et materiaux necessaires ». La Galissonière priait « les officiers et Commandans a Chambly et a $S^{\text {te }}$ Therese de luy [de Léry] donner les habitans, sauvages et voitures dont il pourroit avoir besoin pour les services $\gg .^{3}$ L'ordonnance du 18 avril $1748^{4}$ confirme la précédente.

Arrivé à Saint-Jean, l'officier de Léry met en état les bateaux et les canots qui s'y trouvaient. La Galissonière lui en exprime sa satisfaction. Il lui recommande de communiquer avec le commandant du fort Saint-Frédéric, Charles de Sabrevois, sieur de Bleury, pour réparer une embarcation défectueuse. Les «differens passages de parti » que provoque l'activité de la guerre de frontière font varier le nombre de bateaux et de canots. Le gouverneur avise de Léry de rapporter chaque changement au magasinier qui a reçu l'ordre de tenir un «Registre exprès» des «batteaux et canots du Roi ». Un post-scriptum exprime le souhait que de Léry puisse tirer bon parti de la recrue de Québec.5

Pour aider de Léry à choisir l'emplacement du poste fortifié, La Galissonière divise méthodiquement ses remarques qui rendent compte de la qualité de la construction. Il note tout d'abord

2 AC, C11A, 91: 98-100.

3 Archives de la province de Québec.

4 «Il est ordonné au $\mathrm{S}^{\mathrm{r}}$ de Lery Enseigne en second de se rendre a $\mathrm{S}^{\mathrm{t}}$ Jean Pour y commander pendant la Construction du fort dont il prendra la Direction et ou il Restera jusqu'a nouvel ordre.... Ibid.

5 La Galissonière à de Léry à Saint-Jean, Montréal, 16 mai 1748. Archives de la province de Québec. Pierre-Georges Roy avait lu la « revue de Québec ». Inventaire des papiers de Lery conservés aux Archives de la Province de Québec (3 vol., Québec, 1939-1940), 2: 166. La photocopie de la lettre autographe de La Galissonière indique qu'il s'agit d'une erreur pour la « recrüe de Kébec ». 
que « c'est un grand defaut de n'être qu'a deux toises de l'eau ....». Vis-à-vis le fort, la rivière n'a que six ou sept arpents ; « elle ne doit jamais battre avec violence quelque vent qu'il fasse ». La Galissonière s'occupe de l'effet de l'érosion: «il y a très peu de courant dans cet endroit et que par conséquent ny l'eau ni les glaces ne doivent point emporter les terres et si cela arrivoit a la longue un rang de petits pieux y remedieroit aisément. » Il trouve avantageux de ménager une esplanade de treize toises. On l'a informé que l'inégalité des terrains n'était pas «considérable ». Le gouverneur conseille de corriger cette situation «en prenant des terres soit sur l'esplanade entre le fort et la riviere soit dans le coté le plus haut du fort pour remplir le bas ».

Il est de première importance d'économiser. «Vous devés faire auparavant, écrit La Galissonière à l'officier de Léry, l'estimation de'cette dépense afin de ne la point entreprendre si elle est trop considérable ». Ainsi, faute de ressources financières, le poste fortifié de Saint-Jean sera de construction médiocre:

Il n'est pas mesme necessaire que toute l'enceinte $\mathrm{du}$ fort soit parfaitement de niveau. Il est seulement a souhaiter que les bastions le soient, c'est l'affaire de quelques toises de plus de maçonerie aux fondemens ainsi qu'une bonne partie du dedans. Quand les pieux d'une ou de deux des courtines seroient plus bas il faut s'en consoler pourvu qu'il n'y ait rien en dehors qui vous domine de façon a pouvoir voir dans le fort ce qui le rendroit tout a fait mauvais. ${ }^{6}$

A la suite de l'effondrement des cheminées, ${ }^{7}$ La Galissonière se félicite de «n'avoir pas entrepris tout le fort en pierre ${ }^{8}$

Parallèlement à la direction des travaux de construction, l'officier de Léry a mission de préparer des expéditions militaires. Dans sa dépêche du 17 juillet 1748, La Galissonière s'oppose à la construction d'un hôpital à Saint-Jean: «Il vaut mieux qu'il en coûte quelque chose au roi pour le transport des malades

\footnotetext{
${ }^{6}$ La Galissonière à de Léry, Montréal, 4 juin 1748. Archives de la province de Québec.

7 AC, C11A, $91: 21$

${ }^{8}$ La Galissonière à de Léry, Québec, 10 novembre 1748 . Archives de la province de Québec.
} 
que d'etablir a St Jean un hopital ou ils seroient très mal et en feroient tomber d'autres par imagination ou autrement. ${ }^{9}$

L'édification du fort et l'aménagement de la route devaient être assez avancés à la mi-juillet 1748, puisque le gouverneur en avait reçu de bonnes nouvelles: «M. de Beaujeu M. Varin et toute la comp ${ }^{\text {ie }}$ m'ont dit bien du bien de votre fort ainsi que du chemin. ${ }^{10}$ La Galissonière s'intéresse au déblaiement des rapides; il avise de Léry de communiquer avec l'intendant à ce sujet.

Le 10 août 1748, La Galissonière expédie une lettre d'encouragement à l'officier de Léry: «Si les maladies et la mauvaise volonté des ouvriers vous ont été contraires vous avés été en recompense bien favorisé par les saisons ainsi je compte tousjours que vous en viendrés a votre honneur et que vous mettrés le fort cette automne en etat de loger la Garnison. ${ }^{11}$

Des accusations pèsent sur l'officier de Léry; l'intendant Bigot en est l'instigateur comme l'atteste la lettre du 3 novembre 1748:

Elles [les terres] se payent par voyage de petits demi tombereaux atellé d'un cheval ou il n'y a qu'une poignée de terre. on done par voyage un maron qui vaut deux sols ou plusieurs suivant l'éloignement et lapréciation de l'ingenieur. s'ils étoient donés par gens fidels il n'y auroit que demi mal, mais ils en donent quatre pour un, ou ils les gardent pour eux, et ils les distribuent dans le public et cela fait une augmentation de depense considérable.

il faudroit pour tolerer cette façon de payer etre sur des distributeurs ou que les ingenieurs ne sortissent pas de dessus les terres mais ils n'y vont qu'en passant et rarement, au surplus les tombereaux sont en partie aux officiers et aux ingenieurs même et vous pensés bien qu'on n'examine pas severement si on done deux marons pour un. on paye dans le mesme gout au fort Saint Jean les transports et l'ingenieur a grand soin de fournir les chevaux, on m'en a instruit et on demandera une gratification pour luy [de Léry]. ${ }^{12}$

${ }^{9} I d$. à $I d$., Montréal, 17 juillet 1748. Ibid.

10 Ibid.

11 Québec, 10 août 1748. Ibid.

12 Inventaire des papiers de Léry, 2 : 83-84. 
Le président du Conseil de Marine exprime l'intention de rectifier cette situation dans une dépêche qu'il envoie à La Jonquière et à Bigot, le 11 avril $1749 .{ }^{13}$ Le système de comptabilité manque de rigueur: «Je ne peux avoir l'honneur, écrit Bigot, de vous rendre compte des depenses que l'on fait au fort $S^{t}$ Jean n'en ayant reçu aucun. on dit seulement dans le public qu'il montera a plus de 40 ou $50 \mathrm{~m}$. l. c'est bien de largent pour un fort de piquets. $\gg 14$

La Galissonière avait recommandé aux bons soins du ministre l'officier de Léry: «M. de Lery vous envoie le plan du fort bati a $S^{t}$ Jean. cet ouvrage a été conduit par son fils aîné qui n'en a pas bougé depuis ce printems. Vous savés d'ailleurs que c'est un bon sujet qui a servi avec distinction dans plusieurs partys de guerre et qui en a commandé avec succès. » La Galissonière propose d'accorder à de Léry une gratification dans l'attente d'une promotion. ${ }^{15}$ Le 10 novembre 1748 , il réitère une expression de satisfaction: «M. votre père m'a remis l'état de vos services que je me ferai un plaisir de faire valoir, celui que vous rendés depuis six mois n'est pas oublié $\gg,{ }^{16}$ c'est-à-dire depuis le début de la construction du fort Saint-Jean. Cette même lettre nous apprend que le gouverneur a été «fort content» des plans du fort Saint-Jean. Le «Plan du Fort... au dessus du rapide $\mathrm{S}^{t}$ Jean ou moüille la barque du Lac Champlain » est conservé au dépôt des cartes des Archives Publiques du Canada. ${ }^{17}$ Il porte une indication d'origine: «fait a $\mathrm{S}^{\mathrm{t}}$ Jean le 9 juin 1748. Léry. 》 La Galissonière l'avait approuvé à Montréal, le 10 juin 1748. Le poste fortifié de Saint-Jean fut terminé en mars $1749 .^{18}$

Le 26 mai 1749, La Galissonière confiait à l'officier de Léry la mission de se rendre « au Detroit dans le convoi de M. de Sabrevois $\gg .^{19}$ Le 17 octobre suivant, le gouverneur Pierre-Jacques de

13 Ibid., $2: 86$.

14 Ibid., $2: 82$.

15 Québec, 20 octobre 1748, AC, C11A, 91: 157-158.

16 La Galissonière à de Léry, Québec, 10 novembre 1748. Archives de la province de Québec.

17 H4/950 - Fort Saint-Jean - 1748.

18 Inventaire des papiers de Léry, 2: 91.

19 Ibid., $2: 173$. 
Taffanel, marquis de La Jonquière, ordonnait à de Léry de prendre le commandement de l'artillerie de la colonie en l'absence du chevalier Claude de Beauharnais. ${ }^{20}$

Cette brève étude nous a permis de prendre connaissance de la qualité médiocre de la construction du fort Saint-Jean et de signaler la protection de La Galissonière à l'égard de l'officier de Léry.

Roland Lamontagne, Ph.D.

Faculté des Arts, Université de Montréal.

20 Ibid., 2: 179. - Le 29 janvier 1748, La Galissonière avait ordonné au chevalier Claude de Beauharnais, neveu de Charles de la Boische, marquis de Beauharnais «de partir incessamment de cette ville, pour se rendre dans les Gouvernements de Montréal et des trois-Rivières, et d'y visiter, faire reparer et regler tout ce qui concerne et a raport a la d. artillerie; fait a Quebec le 29 e Janvier $1748 \gg$. Catalogue Nouvelle série no 47 (juin 1960), 4. A la licorne. F. Roux-Devillas, 12 rue Bonaparte, Paris $6^{\circ}$. 